www.revistadyo.com

\title{
Multi-item Inventory Problem: Literature Review and a Proposal for Practitioners
}

Cardós M, de-la-Fuente-Aragón M.V. , Ros-McDonnell L

Recibido: 12 de Enero de 2021

https://doi.org/10.37610/dyo.v0i74.603

Aceptado: 5 de Marzo de 2021

\begin{abstract}
Inventory managers have to assign an inventory policy to each item seeking to satisfy a required global service level with the lowest inventory cost. This paper reviews the existing literature and, based on current research, the most appropriate options are proposed to be used by practitioners.
\end{abstract}

\section{Keywords}

multi-item problem; ABC analysis; VED analysis; ranking index

\section{Introduction}

Almost every company in the distribution industry have to allocate inventory investment among the hundreds or even thousands of items they have to manage. This allocation is critical for a quick response to the customers while maintaining low levels of inventory and operation costs.

Based on a review of the relevant literature on the classification of items, (van Kampen, Akkerman and Pieter van Donk, 2012) propose a conceptual framework considering the aim of the classification (inventory management, forecasting, production strategy), the method applied (characteristics of the items, the technique considered and the resulting categories) and the context (process, product, product life cycle). However, authors claim that their work is "a first step to unravel whether some deeper logic can be found to explain how the different SKU (item) classification decisions are made or should be made ".

Spare parts have been widely studied as reported by (Roda et al., 2014) and (Roda et al., 2012) who highlight the item criticality as an important factor because of the lack of some items may result into tough consequences for the installation. Other authors, e.g. (Andrea Bacchetti and Saccani, 2012), also attribute an important role to the part criticality.

\footnotetext{
mcardos@doe.upv.es

María Victoria de la Fuente Aragón **

marivi.fuente@upct.es

Lorenzo Ross McDonnell **

lorenzo.ros@upct.es

* Dpto. de Organización de Empresas. Universitat

Politécnica de Valencia.

** Industrial Engineering \& Management" Research Group,

Universidad Politécnica de Cartagena
}

Manuel Cardós Carboneras *
However, existing frameworks basically focus on formal aspects of the technique itself but provide scarce insight into important practical questions such as some clues an inventory manager can use to select an appropriate technique. That is precisely the aim of this paper.

This paper is organized as follows. Section 2 presents the most relevant contributions classified according to four different approaches, some of them used extensively by practitioners and others by academics. Section 3 includes an experiment with the most interesting approaches. Finally, section 4 analyses each approach and propose a practical method based on the present knowledge and also concludes on the limitations of the current research.

\section{Literature Review}

\subsection{Direct Determination of the Item Stock Policies}

Different approaches have been developed to cope with the multi-item inventory problem. The first one is the direct determination of the item stock policies in order to minimize a global measure, such as the inventory cost or the average inventory, and sometimes subject to a constraint that usually is expressed as a global service level. In this context, stockout costs and service level are alternative ways to model this problem. (Arrow, Harris and Marschak, 1951) stated that any organization "has a general idea of the value it would attach to the damage that would be caused by the nonavailability of an item". More recently, (van Houtum and Zijm, 2000) describe a general cost model and a general service model and prove that they are equivalent if the penalty cost is proportional to the complementary of the service measure, e.g. the service constraint is the fill rate and the penalty cost is a fixed amount per demand unit that cannot be served directly from the stock. The models are equivalent if the optimal policy for the cost model is also optimal for the 
service model and as a consequence the remaining costs are the same for both models. These results apply to single and multi-stage inventory problems for a single item.

(Mitchell, 1988) copes with the problem of setting up single-item service objectives in a multi-item inventory system subject to a global service level target. He assumes that the demand distribution is logistic and proposes an approach that provides inventory costs $85 \%$ lower than those of the method with identical service levels for all items (named as Identical Service Approach or ISA). He considers that ISA is an approach very used by managers and recommends to replace the logistic distribution by a better approximation to a negative binomial distribution.

Similarly, (Kelle, 1989) proposes four different models of increasing complexity so that approximate solutions have to be considered and with a substantial computational effort.

(Cohen et al., 1992) determine the (s, S) stock policies for every item using an approximate cost function and service level constraint and a greedy heuristic is presented for solving the resulting optimization problem.

(Lenard and Roy, 1995) believe that single-item inventory models are not satisfactory in practice and multi-item models are needed basically because there may be a large number of objectives in inventory control, most of them concerning the overall service level and the average inventory, that are not determined at the item level.

(Thonemann, Brown and Hausman, 2002) analyse multiitem spare parts inventory policies with the objective of minimizing the inventory investment subject to a target fill rate constraint. Two different approaches were compared, the first one using the same target fill rate for every item, and the second one allows different service levels per item but an overall demand-weighted fill rate must accomplish the target fill rate. An approximate model is proposed to quantify the expected improvement when using the second approach.

(Mohammaditabar, Hassan Ghodsypour and Obrien, 2012) propose an integrated model to simultaneously categorize the items and find the best policy, instead of first classifying the items and later determining the stock policies. The resulting problem is so difficult that they use simulated annealing to find a solution.

(De Schrijver, Aghezzaf and Vanmaele, 2013) see a practical need for a system rather than an item approach in a broad range of inventory policies, so that costs may decrease $10 \%$ to $46 \%$ depending on the test cases.

\subsection{ABC Classification using one Criterion}

The second approach focuses on classifying the items into $\mathrm{ABC}$ categories using just one criterion, typically the demand value or the demand volume of the items. Later every item in a category is assigned the same stock policy and service level. In well-known textbooks as (Silver et al. 1998, p.32-35) can be found the foundations and use of the $\mathrm{ABC}$ classification including some guidelines for using inventory policies.

Although the term $\mathrm{ABC}$ suggests the use of three categories, its recommended number and characteristics vary among authors. These variations are explained by (Arcade, 1980) based on the specific form that the Pareto Curve present in different cases, so the ABC categories are not always defined the same way.

(Eilon and Mallya, 1985) highlight the importance of the number and boundaries of the $\mathrm{ABC}$ classes and propose a method to establish them whose required computational effort increases with the number of items considered.

(Zhang, Hopp and Supatgiat, 2001) develop a simple procedure for establishing stock policies for a multiitem distribution centre of spare parts. They formulate the inventory problem in terms of minimizing inventory investment subject to constraints on cycle service level and replenishment frequency. As a result, the first step is to classify the items into classes according to the ratio $\mu_{i} /\left(L_{i} h_{i}{ }^{2}\right)$ where $\mu_{i}$ is the expected demand for item $i$ per year, $L_{i}$ is the leadtime for item $\mathrm{i}$ and $h_{i}$ is the holding cost for item $\mathrm{i}$. The second step is to constrain the cycle service level and order frequency uniformly within each category, so that a search of the optimal service level per category is performed in order to minimize the inventory investment. Probably this is the first time that ABC classes and service levels are determined based on inventory theory.

(Teunter, Babai and Syntetos, 2009) propose a new ABC ranking criterion based on minimizing holding and penalty costs. This cost criterion is the ratio $b_{i} \mu_{i} /\left(h_{i} Q_{i}\right)$ where $b_{i}$ is the unit penalty cost for item $\mathrm{i}, h_{i}$ is the inventory holding cost for item $\mathrm{i}$ and $Q_{i}$ is the order size for item $\mathrm{i}$. Items are classified into categories, the items within a category are assigned the same cycle service level rate and finally its values are determined minimizing inventory costs. Additionally the authors compare their proposed criterion with a modified criterion based on the one proposed by (Zhang, Hopp and Supatgiat, 2001) where the original unit cost is replaced by the inventory holding cost so that the original problem now becomes into minimizing the inventory costs. They claim that their criterion outperforms the one proposed by (Zhang, Hopp and Supatgiat, 2001). 
More recently, (Cardos, Guijarro and Babiloni, 2015) propose minimizing inventory costs subject to service level constraints.

\subsection{Bidimensional Classification}

The third approach is similar to the previous one but using two criteria and a bidimensional classification scheme. Usually the $\mathrm{ABC}$ classification is completed with another classification based on the criticality of the item or on how difficult is to forecast the demand of the item. Usually the items in a category are assigned the same stock policy and service level.

(Ramani and Kutty, 1985) consider that the ABC classification is not appropriate for spare parts because the criticality of the items is ignored. Therefore, they propose a multiple criteria classification combining the $\mathrm{ABC}$ classification and the item criticality (also known as VED that stands for vital, essential and desirable). The nine resulting groups are assigned a service level constraint that decreases in VED and increases in ABC.

(Molenaers et al., 2012) propose a spare part classification based on item criticality including six categories and different factors such as equipment criticality, probability of failure, replenishment time, number of potential suppliers, availability of technical specifications and maintenance type.

\subsection{Multiple Criteria and Techniques}

Finally, the fourth approach uses different criteria and techniques in order to define the classes and to assign every item to a class. Once again, the items in a class share the same stock policy and service level.

(Flores and Whybark, 1988) point out the need of using multiple criteria in classifying the items and the lack of specific guidelines for managing the classification. They identify the dollar-usage criterion as the most usual criterion but also other factors such as item cost, certainty of supply, rate of obsolescence, impact of a stockout of an item, lead time, obsolescence, availability, substitutability and criticality.

(Flores, Olson and Dorai, 1992) propose the use of Analytic Hierarchy Process in order to reduce several criteria into a univariate measure.
(Bhattacharya, Sarkar and Mukherjee, 2007) use of the Technique for Order of Preference by Similarity to Ideal Solution (TOPSIS) in ABC classification, applies the method to a process industry and claim that this methodology contributes to lower the inventory investment.

(Rezaei and Dowlatshahi, 2010) apply a rule-based inference system for classifying inventories into different classes according to their multi-criteria importance.

(Ding and Sun, 2011) propose a multiple criteria classification model that incorporates a linear programming model and a nonlinear model.

(Bacchetti and Saccani, 2012) develop a more complex multi-criteria classification procedure for inventory management based on six dimensions and 12 categories with different forecasting and inventory policies.

(Babai, Ladhari and Lajili, 2015) compare empirically the multi-criteria classification methods proposed by (Ramanathan, 2006), (Zhou and Fan, 2007), (Ng, 2007) and (Hadi-Vencheh, 2010). They recommend the use of the first method given that it outperforms the other ones.

Other techniques are used by other authors such as grouping the items using dynamic programming (Odanaka and Tanaka, 1987), operational constraints (Cohen and Ernst, 1988), artificial intelligence (Yu, 2011), fuzzy AHPDEA (Hadi-Vencheh and Mohamadghasemi, 2011), the rulebased inference system (Rezaei and Dowlatshahi, 2010), the weighted linear optimization (Zhou and Fan, 2007) (Hadi-Vencheh, 2010) (Ramanathan, 2006) (Ng, 2007), the Shannon entropy (Zheng et al., 2017), etc.

\section{Numerical experiment}

\subsection{Data, settings and calculations}

We illustrate the use and performance of some of the ABC ranking approaches using the data-set described in (Flores, Olson and Dorai, 1992) of 47 items on hospital inventory management. Data are shown in Table 1 and have been completed with input from (Babai, Ladhari and Lajili, 2015) on order quantity and standard deviation. These authors also use a unit holding cost of $20 \%$ and model the demand with a normal distribution, which we will maintain for this experiment. In addition, we assume a penalty cost of $30 \%$ and items are managed by an order point stock policy. Note that we use three inventory classes A, B and C with 10, 14 and 23 items respectively. 
Our purpose is to compare different approaches for ordering items and classifying them into categories. Given an item classification, the problem we consider is to determine the optimal service level for each inventory category so that the holding cost is minimal and a global service level is reached. Note that we do not consider the stockout cost because it is replaced by a service constraint. Optimization is performed using Excel Solver.

The strategies we consider are: (a) demand value; (b) demand volume; (c) Zhang criterion; (d) Teunter criterion; and (e) the same service level for each item. Obviously, the last approach is basically for reference.

Calculations are performed as follows. All the items in an inventory class have the same specified target cycle service level (CSL) so we can calculate the reorder point by.

$$
s_{i}=F_{L i}^{-1}(C S L)
$$

Later we calculate the fill rate of each item by

$$
F R_{i}=1-\frac{s_{L i}}{Q_{i}}\left[G\left(\frac{s_{i}-m_{L i}}{s_{L i}}\right)-G\left(\frac{s_{i}-m_{L i}+Q_{i}}{s_{L i}}\right)\right]
$$

and its holding cost by

$$
H C_{i}=h_{i}\left(\frac{Q_{i}}{2}+s_{i}-m_{L i}\right)+h_{i} \frac{s_{L i}^{2}}{Q_{i}}\left[H\left(\frac{s_{i}-m_{L i}}{s_{L i}}\right)-H\left(\frac{s_{i}-m_{L i}+Q_{i}}{s_{L i}}\right)\right]
$$

where

$\mathrm{s}_{\mathrm{i}}=$ reorder point of the $i$-th item,

$\mu_{\mathrm{Li}}=$ average demand of the $i$-th item during its lead time,

$6_{i}=$ standard deviation of the $i$-th item in a time period,

$6_{L i}=$ standard deviation of the $i$-th item during its lead time

$G=$ loss function,

$H=$ Integral of the loss function.

\subsection{Numerical Results}

We have applied the different approaches to the described dataset using the global fill rate as a service constraint. In fact, we have repeated the experiment for global fill rate values of $80 \%, 90 \%, 95 \%$ and $97 \%$.

The results for a global fill rate of $80 \%$ are shown in the tables below. It can be seen that some items move between classes, e.g. item 2 is A class according the demand value approach, B class according the demand volume approach, $\mathrm{C}$ class according the Zhang criterion, and A class according the Teunter criterion. However, only 6 items change class in demand volume, 11 items in Zhang's criteria and 1 item in Teunter's criteria.

It is also remarkable that the CSL for class A is 0.70 while it is 0.72 for Class B with the demand value approach. This can be seen as counterintuitive, but in fact it is an indication that this approach is not what we are looking for. The same comment applies to Teunter's approach. 
Table 1 Dataset used to compare different approaches.

\begin{tabular}{|c|c|c|c|c|c|c|c|}
\hline Item & $\begin{array}{r}\text { Average } \\
\text { unit } \\
\text { cost (\$) }\end{array}$ & $\begin{array}{r}\text { Annual } \\
\text { dollar } \\
\text { demand (\$) }\end{array}$ & $\begin{array}{c}\text { Critical } \\
\text { factor }\end{array}$ & $\begin{array}{r}\text { Lead } \\
\text { time } \\
\text { (weeks) }\end{array}$ & $\begin{array}{r}A B C \\
\text { inventory } \\
\text { class }\end{array}$ & $\begin{array}{r}\text { Ordering } \\
\text { quantity } \\
(E O Q)\end{array}$ & $\begin{array}{l}\text { Std dev } \\
\text { demand } \\
\text { per year }\end{array}$ \\
\hline 1 & 49,92 & $5.840,64$ & 1 & 2 & A & 4,84 & 58,50 \\
\hline 2 & 210,00 & $5.670,00$ & 1 & 5 & A & 1,13 & 13,50 \\
\hline 3 & 23,76 & $5.037,12$ & 1 & 4 & A & 9,45 & 106,00 \\
\hline 4 & 27,73 & $4.769,56$ & 0,01 & 1 & A & 7,88 & 86,00 \\
\hline 5 & 57,98 & $3.478,80$ & 0,5 & 3 & A & 3,22 & 30,00 \\
\hline 6 & 31,24 & $2.936,67$ & 0,5 & 3 & A & 5,49 & 47,00 \\
\hline 7 & 28,20 & $2.820,00$ & 0,5 & 3 & A & 5,96 & 50,00 \\
\hline 8 & 55,00 & $2.640,00$ & 0,01 & 4 & A & 2,95 & 24,00 \\
\hline 9 & 73,44 & $2.423,52$ & 1 & 6 & A & 2,12 & 16,50 \\
\hline 10 & 160,50 & $2.407,50$ & 0,5 & 4 & A & 0,97 & 7,50 \\
\hline 11 & 5,12 & $1.075,20$ & 1 & 2 & B & 20,25 & 105,00 \\
\hline 12 & 20,87 & $1.043,50$ & 0,5 & 5 & B & 4,90 & 25,00 \\
\hline 13 & 86,50 & $1.038,00$ & 1 & 7 & B & 1,18 & 6,00 \\
\hline 14 & 110,40 & 883,20 & 0,5 & 5 & B & 0,85 & 4,00 \\
\hline 15 & 71,20 & 854,40 & 1 & 3 & B & 1,30 & 6,00 \\
\hline 16 & 45,00 & 810,00 & 0,5 & 3 & B & 2,00 & 9,00 \\
\hline 17 & 14,66 & 703,68 & 0,5 & 4 & B & 5,72 & 24,00 \\
\hline 18 & 49,50 & 594,00 & 0,5 & 6 & B & 1,56 & 6,00 \\
\hline 19 & 47,50 & 570,00 & 0,5 & 5 & B & 1,59 & 6,00 \\
\hline 20 & 58,45 & 467,60 & 0,5 & 4 & B & 1,17 & 4,00 \\
\hline 21 & 24,40 & 463,60 & 1 & 4 & B & 2,79 & 9,50 \\
\hline 22 & 65,00 & 455,00 & 0,5 & 4 & B & 1,04 & 3,50 \\
\hline 23 & 86,50 & 432,50 & 1 & 4 & B & 0,76 & 2,50 \\
\hline 24 & 33,20 & 398,40 & 1 & 3 & B & 1,90 & 6,00 \\
\hline 25 & 37,05 & 370,50 & 0,01 & 1 & C & 1,64 & 5,00 \\
\hline 26 & 33,84 & 338,40 & 0,01 & 3 & C & 1,72 & 5,00 \\
\hline 27 & 84,03 & 336,12 & 0,01 & 1 & C & 0,69 & 2,00 \\
\hline 28 & 78,40 & 313,60 & 0,01 & 6 & C & 0,71 & 2,00 \\
\hline 29 & 134,34 & 268,68 & 0,01 & 7 & C & 0,39 & 1,00 \\
\hline 30 & 56,00 & 224,00 & 0,01 & 1 & C & 0,85 & 2,00 \\
\hline 31 & 72,00 & 216,00 & 0,5 & 5 & C & 0,65 & 1,50 \\
\hline 32 & 53,02 & 212,08 & 1 & 2 & C & 0,87 & 2,00 \\
\hline 33 & 49,48 & 197,92 & 0,01 & 5 & C & 0,90 & 2,00 \\
\hline 34 & 7,07 & 190,89 & 0,01 & 7 & C & 6,18 & 13,50 \\
\hline 35 & 60,60 & 181,80 & 0,01 & 3 & C & 0,70 & 1,50 \\
\hline 36 & 40,82 & 163,28 & 1 & 3 & C & 0,99 & 2,00 \\
\hline 37 & 30,00 & 150,00 & 0,01 & 5 & C & 1,29 & 2,50 \\
\hline 38 & 67,40 & 134,80 & 0,5 & 3 & C & 0,55 & 1,00 \\
\hline 39 & 59,60 & 119,20 & 0,01 & 5 & C & 0,58 & 1,00 \\
\hline 40 & 51,68 & 103,36 & 0,01 & 6 & C & 0,62 & 1,00 \\
\hline 41 & 19,80 & 79,20 & 0,01 & 2 & C & 1,42 & 2,00 \\
\hline 42 & 37,70 & 75,40 & 0,01 & 2 & C & 0,73 & 1,00 \\
\hline 43 & 29,89 & 59,78 & 0,01 & 5 & C & 0,82 & 1,00 \\
\hline 44 & 48,30 & 48,30 & 0,01 & 3 & C & 0,46 & 0,50 \\
\hline 45 & 34,40 & 34,40 & 0,01 & 7 & C & 0,54 & 0,50 \\
\hline 46 & 28,80 & 28,80 & 0,01 & 3 & C & 0,59 & 0,50 \\
\hline 47 & 8,46 & 25,38 & 0,01 & 5 & C & 1,88 & 1,50 \\
\hline
\end{tabular}


Table 2 Every item is assigned the same cycle service level (Equal approach).

Annual $\quad A B C$

dollar inventory Category $\quad S_{i} \quad S_{i} \quad$ Holding Item demand (\$) class CSL (units) (units) CSL $L_{i} \quad F R_{i}$ cost

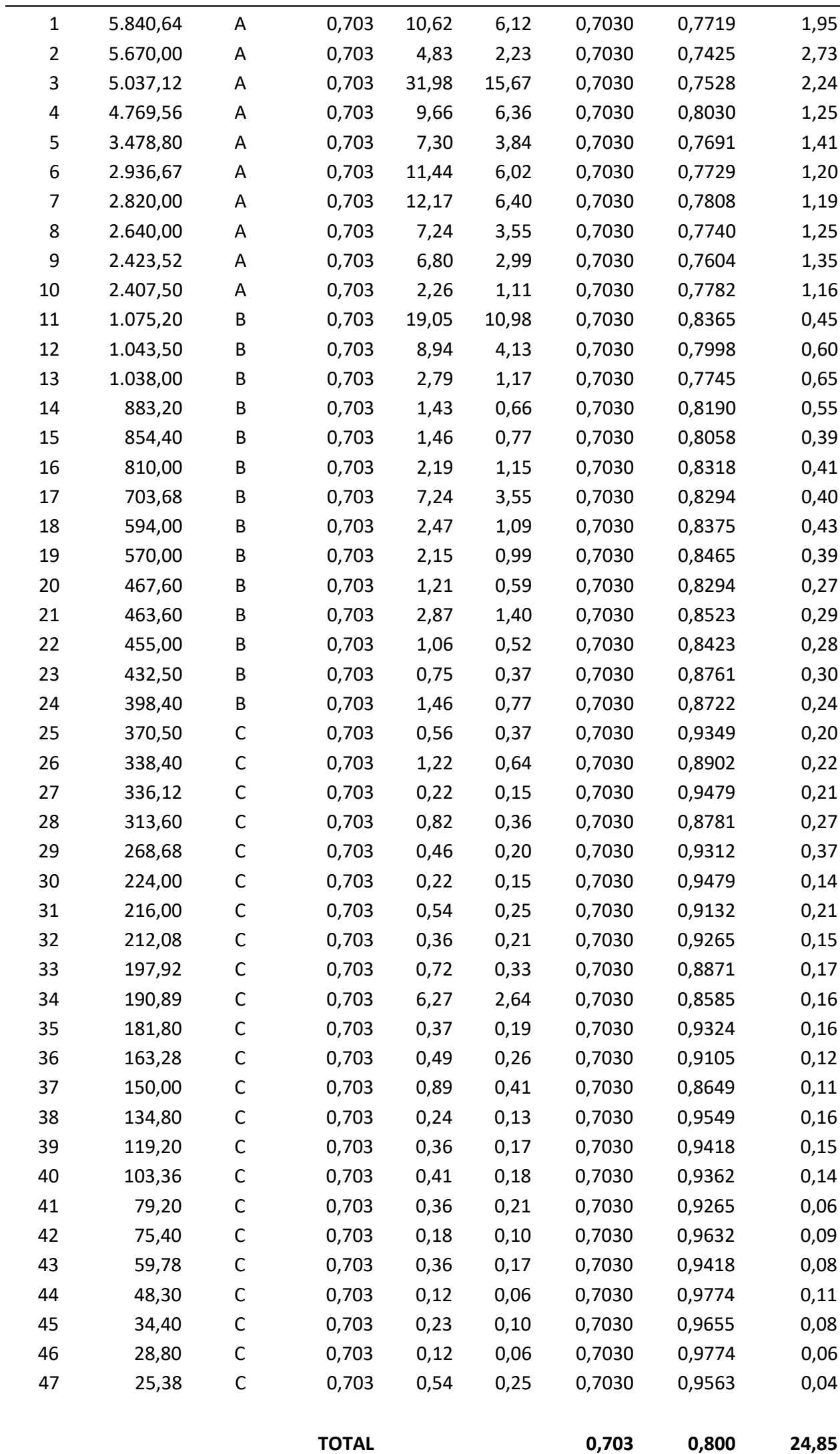


Table 3 Items are ranked using the value of demand (Demand value approach).

\begin{tabular}{|c|c|c|c|c|c|c|c|c|}
\hline Item & $\begin{array}{r}\text { Annual } \\
\text { dollar } \\
\text { demand (\$) }\end{array}$ & $\begin{array}{r}A B C \\
\text { inventory } \\
\text { class }\end{array}$ & $\begin{array}{r}\text { Category } \\
\text { CSL }\end{array}$ & $\begin{array}{r}S_{i} \\
\text { (units) }\end{array}$ & $\begin{array}{r}S_{S_{i}} \\
\text { (units) }\end{array}$ & $C S L_{i}$ & $F R_{i}$ & $\begin{array}{r}\text { Holding } \\
\text { cost }\end{array}$ \\
\hline 1 & $5.840,64$ & A & 0,70 & 10,57 & 6,07 & 0,7017 & 0,7707 & 1,94 \\
\hline 2 & $5.670,00$ & A & 0,70 & 4,81 & 2,22 & 0,7017 & 0,7412 & 2,72 \\
\hline 3 & $5.037,12$ & $A$ & 0,70 & 31,87 & 15,56 & 0,7017 & 0,7516 & 2,23 \\
\hline 4 & $4.769,56$ & A & 0,70 & 9,62 & 6,31 & 0,7017 & 0,8020 & 1,24 \\
\hline 5 & $3.478,80$ & $A$ & 0,70 & 7,28 & 3,81 & 0,7017 & 0,7680 & 1,40 \\
\hline 6 & $2.936,67$ & A & 0,70 & 11,40 & 5,97 & 0,7017 & 0,7717 & 1,20 \\
\hline 7 & $2.820,00$ & $A$ & 0,70 & 12,13 & 6,36 & 0,7017 & 0,7797 & 1,18 \\
\hline 8 & $2.640,00$ & A & 0,70 & 7,22 & 3,52 & 0,7017 & 0,7729 & 1,25 \\
\hline 9 & $2.423,52$ & A & 0,70 & 6,77 & 2,97 & 0,7017 & 0,7593 & 1,35 \\
\hline 10 & $2.407,50$ & A & 0,70 & 2,25 & 1,10 & 0,7017 & 0,7771 & 1,15 \\
\hline 11 & $1.075,20$ & B & 0,76 & 22,68 & 14,60 & 0,7609 & 0,8749 & 0,51 \\
\hline 12 & $1.043,50$ & B & 0,76 & 10,31 & 5,50 & 0,7609 & 0,8447 & 0,69 \\
\hline 13 & $1.038,00$ & B & 0,76 & 3,18 & 1,56 & 0,7609 & 0,8234 & 0,76 \\
\hline 14 & 883,20 & B & 0,76 & 1,65 & 0,88 & 0,7609 & 0,8606 & 0,62 \\
\hline 15 & 854,40 & B & 0,76 & 1,71 & 1,02 & 0,7609 & 0,8498 & 0,45 \\
\hline 16 & 810,00 & B & 0,76 & 2,57 & 1,53 & 0,7609 & 0,8711 & 0,46 \\
\hline 17 & 703,68 & B & 0,76 & 8,41 & 4,72 & 0,7609 & 0,8691 & 0,46 \\
\hline 18 & 594,00 & B & 0,76 & 2,83 & 1,45 & 0,7609 & 0,8757 & 0,49 \\
\hline 19 & 570,00 & B & 0,76 & 2,47 & 1,32 & 0,7609 & 0,8830 & 0,44 \\
\hline 20 & 467,60 & B & 0,76 & 1,40 & 0,79 & 0,7609 & 0,8691 & 0,31 \\
\hline 21 & 463,60 & B & 0,76 & 3,33 & 1,87 & 0,7609 & 0,8876 & 0,33 \\
\hline 22 & 455,00 & B & 0,76 & 1,23 & 0,69 & 0,7609 & 0,8796 & 0,31 \\
\hline 23 & 432,50 & B & 0,76 & 0,88 & 0,49 & 0,7609 & 0,9064 & 0,34 \\
\hline 24 & 398,40 & B & 0,76 & 1,71 & 1,02 & 0,7609 & 0,9033 & 0,27 \\
\hline 25 & 370,50 & C & 0,40 & 0,02 & $-0,17$ & 0,4032 & 0,8155 & 0,13 \\
\hline 26 & 338,40 & C & 0,40 & 0,28 & $-0,29$ & 0,4032 & 0,7007 & 0,12 \\
\hline 27 & 336,12 & C & 0,40 & 0,01 & $-0,07$ & 0,4032 & 0,8521 & 0,15 \\
\hline 28 & 313,60 & C & 0,40 & 0,30 & $-0,17$ & 0,4032 & 0,6737 & 0,15 \\
\hline 29 & 268,68 & C & 0,40 & 0,18 & $-0,09$ & 0,4032 & 0,8051 & 0,24 \\
\hline 30 & 224,00 & C & 0,40 & 0,01 & $-0,07$ & 0,4032 & 0,8521 & 0,10 \\
\hline 31 & 216,00 & C & 0,40 & 0,17 & $-0,11$ & 0,4032 & 0,7570 & 0,13 \\
\hline 32 & 212,08 & C & 0,40 & 0,06 & $-0,10$ & 0,4032 & 0,7922 & 0,09 \\
\hline 33 & 197,92 & C & 0,40 & 0,23 & $-0,15$ & 0,4032 & 0,6936 & 0,09 \\
\hline 34 & 190,89 & C & 0,40 & 2,42 & $-1,21$ & 0,4032 & 0,6330 & 0,08 \\
\hline 35 & 181,80 & C & 0,40 & 0,08 & $-0,09$ & 0,4032 & 0,8085 & 0,11 \\
\hline 36 & 163,28 & C & 0,40 & 0,11 & $-0,12$ & 0,4032 & 0,7500 & 0,07 \\
\hline 37 & 150,00 & C & 0,40 & 0,29 & $-0,19$ & 0,4032 & 0,6457 & 0,06 \\
\hline 38 & 134,80 & C & 0,40 & 0,06 & $-0,06$ & 0,4032 & 0,8719 & 0,12 \\
\hline 39 & 119,20 & C & 0,40 & 0,12 & $-0,08$ & 0,4032 & 0,8347 & 0,11 \\
\hline 40 & 103,36 & C & 0,40 & 0,15 & $-0,08$ & 0,4032 & 0,8192 & 0,09 \\
\hline 41 & 79,20 & C & 0,40 & 0,06 & $-0,10$ & 0,4032 & 0,7922 & 0,04 \\
\hline 42 & 75,40 & C & 0,40 & 0,03 & $-0,05$ & 0,4032 & 0,8954 & 0,07 \\
\hline 43 & 59,78 & C & 0,40 & 0,12 & $-0,08$ & 0,4032 & 0,8347 & 0,05 \\
\hline 44 & 48,30 & C & 0,40 & 0,03 & $-0,03$ & 0,4032 & 0,9359 & 0,09 \\
\hline 45 & 34,40 & C & 0,40 & 0,09 & $-0,04$ & 0,4032 & 0,9021 & 0,06 \\
\hline 46 & 28,80 & C & 0,40 & 0,03 & $-0,03$ & 0,4032 & 0,9359 & 0,05 \\
\hline 47 & 25,38 & C & 0,40 & 0,17 & $-0,11$ & 0,4032 & 0,8759 & 0,03 \\
\hline & & & TOTAL & & & 0,698 & 0,800 & 24,35 \\
\hline
\end{tabular}


Table 4 Items are ranked using the units demanded (Demand volume approach).

Annual $\quad A B C$

demand inventory Category $\quad s_{i} \quad S_{i} \quad$ Holding Item (unit/year) class CSL (units) (units) CSL $L_{i} \quad F R_{i}$ cost

\begin{tabular}{|c|c|c|c|c|c|c|c|c|}
\hline 3 & 212 & $A$ & 0,81 & 42,21 & 25,90 & 0,8108 & 0,8485 & 2,99 \\
\hline 11 & 210 & $A$ & 0,81 & 26,22 & 18,14 & 0,8108 & 0,9058 & 0,57 \\
\hline 4 & 172 & $A$ & 0,81 & 13,81 & 10,51 & 0,8108 & 0,8838 & 1,62 \\
\hline 1 & 117 & $A$ & 0,81 & 14,61 & 10,11 & 0,8108 & 0,8622 & 2,58 \\
\hline 7 & 100 & $A$ & 0,81 & 16,35 & 10,58 & 0,8108 & 0,8684 & 1,56 \\
\hline 6 & 94 & $A$ & 0,81 & 15,37 & 9,95 & 0,8108 & 0,8629 & 1,59 \\
\hline 5 & 60 & $A$ & 0,81 & 9,81 & 6,35 & 0,8108 & 0,8602 & 1,87 \\
\hline 12 & 50 & $A$ & 0,81 & 11,64 & 6,83 & 0,8108 & 0,8816 & 0,79 \\
\hline 8 & 48 & $A$ & 0,81 & 9,56 & 5,86 & 0,8108 & 0,8637 & 1,66 \\
\hline 17 & 48 & $A$ & 0,81 & 9,56 & 5,86 & 0,8108 & 0,9012 & 0,52 \\
\hline 9 & 33 & B & 0,34 & 1,51 & $-2,30$ & 0,3409 & 0,4089 & 0,47 \\
\hline 2 & 27 & B & 0,34 & 0,88 & $-1,72$ & 0,3409 & 0,3859 & 0,92 \\
\hline 34 & 27 & B & 0,34 & 1,60 & $-2,03$ & 0,3409 & 0,5731 & 0,07 \\
\hline 21 & 19 & B & 0,34 & 0,38 & $-1,08$ & 0,3409 & 0,5600 & 0,12 \\
\hline 16 & 18 & B & 0,34 & 0,15 & $-0,89$ & 0,3409 & 0,5202 & 0,16 \\
\hline 10 & 15 & B & 0,34 & 0,30 & $-0,85$ & 0,3409 & 0,4332 & 0,42 \\
\hline 13 & 12 & B & 0,34 & 0,71 & $-0,90$ & 0,3409 & 0,4280 & 0,23 \\
\hline 15 & 12 & B & 0,34 & 0,10 & $-0,59$ & 0,3409 & 0,4753 & 0,15 \\
\hline 18 & 12 & $B$ & 0,34 & 0,55 & $-0,84$ & 0,3409 & 0,5309 & 0,18 \\
\hline 19 & 12 & B & 0,34 & 0,39 & $-0,76$ & 0,3409 & 0,5484 & 0,16 \\
\hline 24 & 12 & $B$ & 0,34 & 0,10 & $-0,59$ & 0,3409 & 0,6036 & 0,11 \\
\hline 25 & 10 & B & 0,34 & $-0,09$ & $-0,28$ & 0,3409 & 0,7799 & 0,12 \\
\hline 26 & 10 & $B$ & 0,34 & 0,08 & $-0,49$ & 0,3409 & 0,6475 & 0,11 \\
\hline 14 & 8 & B & 0,34 & 0,26 & $-0,51$ & 0,3409 & 0,4973 & 0,21 \\
\hline 20 & 8 & $C$ & 0,21 & $-0,26$ & $-0,88$ & 0,2148 & 0,3714 & 0,07 \\
\hline 22 & 7 & $C$ & 0,21 & $-0,23$ & $-0,77$ & 0,2148 & 0,3962 & 0,07 \\
\hline 23 & 5 & $C$ & 0,21 & $-0,16$ & $-0,55$ & 0,2148 & 0,4747 & 0,09 \\
\hline 37 & 5 & $C$ & 0,21 & $-0,13$ & $-0,61$ & 0,2148 & 0,4461 & 0,03 \\
\hline 27 & 4 & $C$ & 0,21 & $-0,14$ & $-0,22$ & 0,2148 & 0,7472 & 0,11 \\
\hline 28 & 4 & $C$ & 0,21 & $-0,08$ & $-0,54$ & 0,2148 & 0,4803 & 0,08 \\
\hline 30 & 4 & $C$ & 0,21 & $-0,14$ & $-0,22$ & 0,2148 & 0,7472 & 0,07 \\
\hline 32 & 4 & $C$ & 0,21 & $-0,16$ & $-0,31$ & 0,2148 & 0,6483 & 0,06 \\
\hline 33 & 4 & $C$ & 0,21 & $-0,11$ & $-0,49$ & 0,2148 & 0,5058 & 0,05 \\
\hline 36 & 4 & $C$ & 0,21 & $-0,15$ & $-0,38$ & 0,2148 & 0,5840 & 0,05 \\
\hline 41 & 4 & $C$ & 0,21 & $-0,16$ & $-0,31$ & 0,2148 & 0,6483 & 0,02 \\
\hline 31 & 3 & $C$ & 0,21 & $-0,08$ & $-0,37$ & 0,2148 & 0,5943 & 0,08 \\
\hline 35 & 3 & $C$ & 0,21 & $-0,11$ & $-0,28$ & 0,2148 & 0,6745 & 0,07 \\
\hline 47 & 3 & $C$ & 0,21 & $-0,08$ & $-0,37$ & 0,2148 & 0,7878 & 0,02 \\
\hline 29 & 2 & $C$ & 0,21 & $-0,02$ & $-0,29$ & 0,2148 & 0,6690 & 0,16 \\
\hline 38 & 2 & $C$ & 0,21 & $-0,07$ & $-0,19$ & 0,2148 & 0,7809 & 0,09 \\
\hline 39 & 2 & $C$ & 0,21 & $-0,05$ & $-0,24$ & 0,2148 & 0,7179 & 0,08 \\
\hline 40 & 2 & $C$ & 0,21 & $-0,04$ & $-0,27$ & 0,2148 & 0,6920 & 0,06 \\
\hline 42 & 2 & $C$ & 0,21 & $-0,08$ & $-0,15$ & 0,2148 & 0,8211 & 0,05 \\
\hline 43 & 2 & $C$ & 0,21 & $-0,05$ & $-0,24$ & 0,2148 & 0,7179 & 0,04 \\
\hline 44 & 1 & $C$ & 0,21 & $-0,04$ & $-0,09$ & 0,2148 & 0,8904 & 0,08 \\
\hline 45 & 1 & $C$ & 0,21 & $-0,01$ & $-0,14$ & 0,2148 & 0,8326 & 0,05 \\
\hline 46 & 1 & $C$ & 0,21 & $-0,04$ & $-0,09$ & 0,2148 & 0,8904 & 0,05 \\
\hline \multicolumn{6}{|c|}{ TOTAL } & 0,703 & 0,800 & 0,709 \\
\hline
\end{tabular}


Table 5 Items are ranked using Zhang criterion (Zhang approach).

\begin{tabular}{|c|c|c|c|c|c|c|c|c|}
\hline Item & $\begin{array}{r}\text { Zhang } \\
\text { index }\end{array}$ & $\begin{array}{r}A B C \\
\text { inventory } \\
\text { class }\end{array}$ & $\begin{array}{r}\text { Category } \\
\text { CSL }\end{array}$ & $\begin{array}{r}s_{i} \\
\text { (units) }\end{array}$ & $\begin{array}{r}S_{i} \\
\text { (units) }\end{array}$ & $C S L_{i}$ & $F R_{i}$ & $\begin{array}{r}\text { holding } \\
\text { cost }\end{array}$ \\
\hline 11 & 5207,06 & A & 0,84 & 28,39 & 20,31 & 0,8380 & 0,9218 & 0,61 \\
\hline 4 & 290,79 & A & 0,84 & 15,07 & 11,76 & 0,8380 & 0,9028 & 1,74 \\
\hline 3 & 122,05 & A & 0,84 & 45,31 & 29,00 & 0,8380 & 0,8718 & 3,23 \\
\hline 34 & 100,32 & A & 0,84 & 8,52 & 4,89 & 0,8380 & 0,9335 & 0,22 \\
\hline 17 & 72,59 & A & 0,84 & 10,26 & 6,57 & 0,8380 & 0,9178 & 0,55 \\
\hline 7 & 54,49 & A & 0,84 & 17,62 & 11,85 & 0,8380 & 0,8894 & 1,68 \\
\hline 6 & 41,74 & A & 0,84 & 16,56 & 11,14 & 0,8380 & 0,8846 & 1,72 \\
\hline 1 & 30,52 & A & 0,84 & 15,82 & 11,32 & 0,8380 & 0,8839 & 2,78 \\
\hline 12 & 29,85 & A & 0,84 & 12,45 & 7,65 & 0,8380 & 0,9009 & 0,84 \\
\hline 47 & 10,90 & A & 0,84 & 0,75 & 0,46 & 0,8380 & 0,9801 & 0,05 \\
\hline 21 & 10,37 & B & 0,53 & 1,67 & 0,21 & 0,5313 & 0,7306 & 0,20 \\
\hline 25 & 9,47 & B & 0,53 & 0,25 & 0,05 & 0,5313 & 0,8750 & 0,16 \\
\hline 5 & 7,73 & B & 0,53 & 4,03 & 0,57 & 0,5313 & 0,6121 & 0,90 \\
\hline 41 & 6,63 & B & 0,53 & 0,18 & 0,03 & 0,5313 & 0,8590 & 0,04 \\
\hline 8 & 5,16 & B & 0,53 & 4,22 & 0,52 & 0,5313 & 0,6184 & 0,81 \\
\hline 24 & 4,72 & B & 0,53 & 0,81 & 0,11 & 0,5313 & 0,7628 & 0,17 \\
\hline 16 & 3,85 & B & 0,53 & 1,21 & 0,17 & 0,5313 & 0,6993 & 0,28 \\
\hline 26 & 3,78 & B & 0,53 & 0,67 & 0,09 & 0,5313 & 0,7932 & 0,16 \\
\hline 30 & 1,66 & B & 0,53 & 0,10 & 0,02 & 0,5313 & 0,8999 & 0,12 \\
\hline 37 & 1,44 & B & 0,53 & 0,54 & 0,06 & 0,5313 & 0,7507 & 0,08 \\
\hline 19 & 1,38 & B & 0,53 & 1,30 & 0,15 & 0,5313 & 0,7216 & 0,27 \\
\hline 9 & 1,33 & B & 0,53 & 4,25 & 0,44 & 0,5313 & 0,6009 & 0,86 \\
\hline 18 & 1,06 & B & 0,53 & 1,54 & 0,16 & 0,5313 & 0,7079 & 0,29 \\
\hline 36 & 1,04 & B & 0,53 & 0,27 & 0,04 & 0,5313 & 0,8293 & 0,09 \\
\hline 15 & 1,03 & C & 0,10 & $-1,15$ & $-1,85$ & 0,1001 & 0,1799 & 0,04 \\
\hline 32 & 0,92 & C & 0,10 & $-0,35$ & $-0,50$ & 0,1001 & 0,4981 & 0,04 \\
\hline 42 & 0,91 & C & 0,10 & $-0,17$ & $-0,25$ & 0,1001 & 0,7395 & 0,04 \\
\hline 20 & 0,76 & C & 0,10 & $-0,81$ & $-1,42$ & 0,1001 & 0,2110 & 0,03 \\
\hline 27 & 0,74 & C & 0,10 & $-0,28$ & $-0,36$ & 0,1001 & 0,6325 & 0,08 \\
\hline 43 & 0,58 & C & 0,10 & $-0,20$ & $-0,40$ & 0,1001 & 0,5911 & 0,03 \\
\hline 22 & 0,54 & C & 0,10 & $-0,71$ & $-1,24$ & 0,1001 & 0,2316 & 0,03 \\
\hline 46 & 0,52 & C & 0,10 & $-0,10$ & $-0,15$ & 0,1001 & 0,8405 & 0,04 \\
\hline 33 & 0,42 & C & 0,10 & $-0,41$ & $-0,79$ & 0,1001 & 0,3343 & 0,03 \\
\hline 35 & 0,35 & C & 0,10 & $-0,29$ & $-0,46$ & 0,1001 & 0,5321 & 0,05 \\
\hline 13 & 0,30 & C & 0,10 & $-1,20$ & $-2,82$ & 0,1001 & 0,1480 & 0,06 \\
\hline 23 & 0,22 & C & 0,10 & $-0,50$ & $-0,89$ & 0,1001 & 0,3032 & 0,05 \\
\hline 38 & 0,19 & C & 0,10 & $-0,19$ & $-0,31$ & 0,1001 & 0,6811 & 0,07 \\
\hline 10 & 0,19 & C & 0,10 & $-1,51$ & $-2,66$ & 0,1001 & 0,1513 & 0,10 \\
\hline 44 & 0,19 & C & 0,10 & $-0,10$ & $-0,15$ & 0,1001 & 0,8405 & 0,07 \\
\hline 14 & 0,17 & C & 0,10 & $-0,82$ & $-1,59$ & 0,1001 & 0,1963 & 0,06 \\
\hline 40 & 0,16 & C & 0,10 & $-0,20$ & $-0,44$ & 0,1001 & 0,5556 & 0,04 \\
\hline 2 & 0,16 & C & 0,10 & $-2,77$ & $-5,36$ & 0,1001 & 0,1233 & 0,21 \\
\hline 45 & 0,16 & C & 0,10 & $-0,10$ & $-0,23$ & 0,1001 & 0,7563 & 0,04 \\
\hline 31 & 0,15 & C & 0,10 & $-0,31$ & $-0,60$ & 0,1001 & 0,4316 & 0,05 \\
\hline 39 & 0,15 & C & 0,10 & $-0,20$ & $-0,40$ & 0,1001 & 0,5911 & 0,05 \\
\hline 28 & 0,14 & C & 0,10 & $-0,41$ & $-0,87$ & 0,1001 & 0,3086 & 0,04 \\
\hline 29 & 0,02 & C & 0,10 & $-0,20$ & $-0,47$ & 0,1001 & 0,5248 & 0,11 \\
\hline & & TOTAL & & & & 0,715 & 0,800 & 19,210 \\
\hline
\end{tabular}


Table 6 Items are ranked using Teunter criterion (Teunter approach).
$\mathrm{ABC}$

\begin{tabular}{|c|c|c|c|c|c|c|c|c|}
\hline Item & $\begin{array}{r}\text { Teunter } \\
\text { index }\end{array}$ & $\begin{array}{r}\text { inventory } \\
\text { class }\end{array}$ & $\begin{array}{r}\text { Category } \\
\text { CSL }\end{array}$ & $\begin{array}{r}S_{i} \\
\text { (units) }\end{array}$ & $\begin{array}{r}S_{\mathrm{i}} \\
\text { (units) }\end{array}$ & $C S L_{i}$ & $F R_{i}$ & $\begin{array}{r}\text { holding } \\
\text { cost }\end{array}$ \\
\hline 2 & 40,50 & $A$ & 0,70 & 4,80 & 2,21 & 0,7010 & 0,7406 & 2,72 \\
\hline 3 & 35,33 & $A$ & 0,70 & 31,81 & 15,50 & 0,7010 & 0,7510 & 2,23 \\
\hline 1 & 35,10 & $A$ & 0,70 & 10,55 & 6,05 & 0,7010 & 0,7702 & 1,94 \\
\hline 4 & 32,25 & $A$ & 0,70 & 9,60 & 6,29 & 0,7010 & 0,8015 & 1,24 \\
\hline 5 & 30,00 & $A$ & 0,70 & 7,26 & 3,80 & 0,7010 & 0,7674 & 1,40 \\
\hline 6 & 28,20 & $A$ & 0,70 & 11,38 & 5,95 & 0,7010 & 0,7712 & 1,20 \\
\hline 7 & 25,00 & $A$ & 0,70 & 12,10 & 6,33 & 0,7010 & 0,7791 & 1,18 \\
\hline 9 & 24,75 & A & 0,70 & 6,76 & 2,96 & 0,7010 & 0,7587 & 1,34 \\
\hline 8 & 24,00 & $A$ & 0,70 & 7,20 & 3,51 & 0,7010 & 0,7723 & 1,25 \\
\hline 10 & 22,50 & $A$ & 0,70 & 2,25 & 1,10 & 0,7010 & 0,7765 & 1,15 \\
\hline 15 & 18,00 & B & 0,77 & 1,76 & 1,06 & 0,7700 & 0,8564 & 0,46 \\
\hline 13 & 18,00 & B & 0,77 & 3,24 & 1,63 & 0,7700 & 0,8309 & 0,77 \\
\hline 11 & 15,75 & $B$ & 0,77 & 23,29 & 15,21 & 0,7700 & 0,8807 & 0,52 \\
\hline 12 & 15,00 & $B$ & 0,77 & 10,54 & 5,73 & 0,7700 & 0,8516 & 0,71 \\
\hline 16 & 13,50 & $B$ & 0,77 & 2,64 & 1,60 & 0,7700 & 0,8770 & 0,47 \\
\hline 20 & 12,00 & $B$ & 0,77 & 1,44 & 0,82 & 0,7700 & 0,8751 & 0,31 \\
\hline 14 & 12,00 & $B$ & 0,77 & 1,69 & 0,92 & 0,7700 & 0,8669 & 0,64 \\
\hline 17 & 12,00 & $B$ & 0,77 & 8,61 & 4,92 & 0,7700 & 0,8751 & 0,47 \\
\hline 22 & 10,50 & $B$ & 0,77 & 1,26 & 0,72 & 0,7700 & 0,8852 & 0,32 \\
\hline 21 & 9,50 & $B$ & 0,77 & 3,41 & 1,95 & 0,7700 & 0,8929 & 0,34 \\
\hline 19 & 9,00 & $B$ & 0,77 & 2,53 & 1,37 & 0,7700 & 0,8884 & 0,45 \\
\hline 18 & 9,00 & $B$ & 0,77 & 2,89 & 1,51 & 0,7700 & 0,8815 & 0,50 \\
\hline 24 & 9,00 & $B$ & 0,77 & 1,76 & 1,06 & 0,7700 & 0,9080 & 0,27 \\
\hline 37 & 7,50 & $B$ & 0,77 & 1,05 & 0,57 & 0,7700 & 0,9024 & 0,13 \\
\hline 23 & 7,50 & C & 0,37 & 0,16 & $-0,23$ & 0,3726 & 0,6422 & 0,15 \\
\hline 25 & 7,50 & C & 0,37 & $-0,03$ & $-0,23$ & 0,3726 & 0,7987 & 0,12 \\
\hline 26 & 7,50 & C & 0,37 & 0,19 & $-0,39$ & 0,3726 & 0,6754 & 0,12 \\
\hline 34 & 6,75 & C & 0,37 & 2,03 & $-1,61$ & 0,3726 & 0,6043 & 0,08 \\
\hline 41 & 6,00 & C & 0,37 & 0,03 & $-0,13$ & 0,3726 & 0,7734 & 0,03 \\
\hline 32 & 6,00 & C & 0,37 & 0,03 & $-0,13$ & 0,3726 & 0,7734 & 0,09 \\
\hline 30 & 6,00 & C & 0,37 & $-0,01$ & $-0,09$ & 0,3726 & 0,8385 & 0,10 \\
\hline 36 & 6,00 & C & 0,37 & 0,07 & $-0,16$ & 0,3726 & 0,7279 & 0,07 \\
\hline 27 & 6,00 & C & 0,37 & $-0,01$ & $-0,09$ & 0,3726 & 0,8385 & 0,14 \\
\hline 33 & 6,00 & C & 0,37 & 0,18 & $-0,20$ & 0,3726 & 0,6679 & 0,09 \\
\hline 28 & 6,00 & C & 0,37 & 0,24 & $-0,22$ & 0,3726 & 0,6469 & 0,14 \\
\hline 35 & 4,50 & C & 0,37 & 0,06 & $-0,12$ & 0,3726 & 0,7911 & 0,10 \\
\hline 31 & 4,50 & $C$ & 0,37 & 0,14 & $-0,15$ & 0,3726 & 0,7354 & 0,12 \\
\hline 42 & 3,00 & C & 0,37 & 0,01 & $-0,06$ & 0,3726 & 0,8858 & 0,07 \\
\hline 38 & 3,00 & C & 0,37 & 0,04 & $-0,08$ & 0,3726 & 0,8601 & 0,12 \\
\hline 40 & 3,00 & C & 0,37 & 0,12 & $-0,11$ & 0,3726 & 0,8027 & 0,09 \\
\hline 43 & 3,00 & $\mathrm{C}$ & 0,37 & 0,09 & $-0,10$ & 0,3726 & 0,8196 & 0,05 \\
\hline 39 & 3,00 & C & 0,37 & 0,09 & $-0,10$ & 0,3726 & 0,8196 & 0,10 \\
\hline 29 & 3,00 & C & 0,37 & 0,15 & $-0,12$ & 0,3726 & 0,7874 & 0,23 \\
\hline 47 & 2,25 & C & 0,37 & 0,14 & $-0,15$ & 0,3726 & 0,8646 & 0,03 \\
\hline 46 & 1,50 & C & 0,37 & 0,02 & $-0,04$ & 0,3726 & 0,9301 & 0,05 \\
\hline 44 & 1,50 & C & 0,37 & 0,02 & $-0,04$ & 0,3726 & 0,9301 & 0,09 \\
\hline 45 & 1,50 & C & 0,37 & 0,08 & $-0,06$ & 0,3726 & 0,8932 & 0,06 \\
\hline TAL & & & & & & 0,6981 & 0,7999 & 24,227 \\
\hline
\end{tabular}




\subsection{Experiment Summary}

Figure 1 indicates, not only that costs increase with FR, but that only demand volume and Zhang show significant reductions in costs. Figure 2 shows the cost reduction of each approach with respect to the equal approach.
This result contradicts Teunter's claim, but there are some reasons that justify this result. The first one is that these items are slow-moving and consequently the approximations used by (Teunter, Babai and Syntetos, 2009) may fail in their CSL estimation. But the most important reason may be that they use stockout costs in a context where they have been replaced by a service constraint.

Figure 1 Holding cost vs. Fill rate.

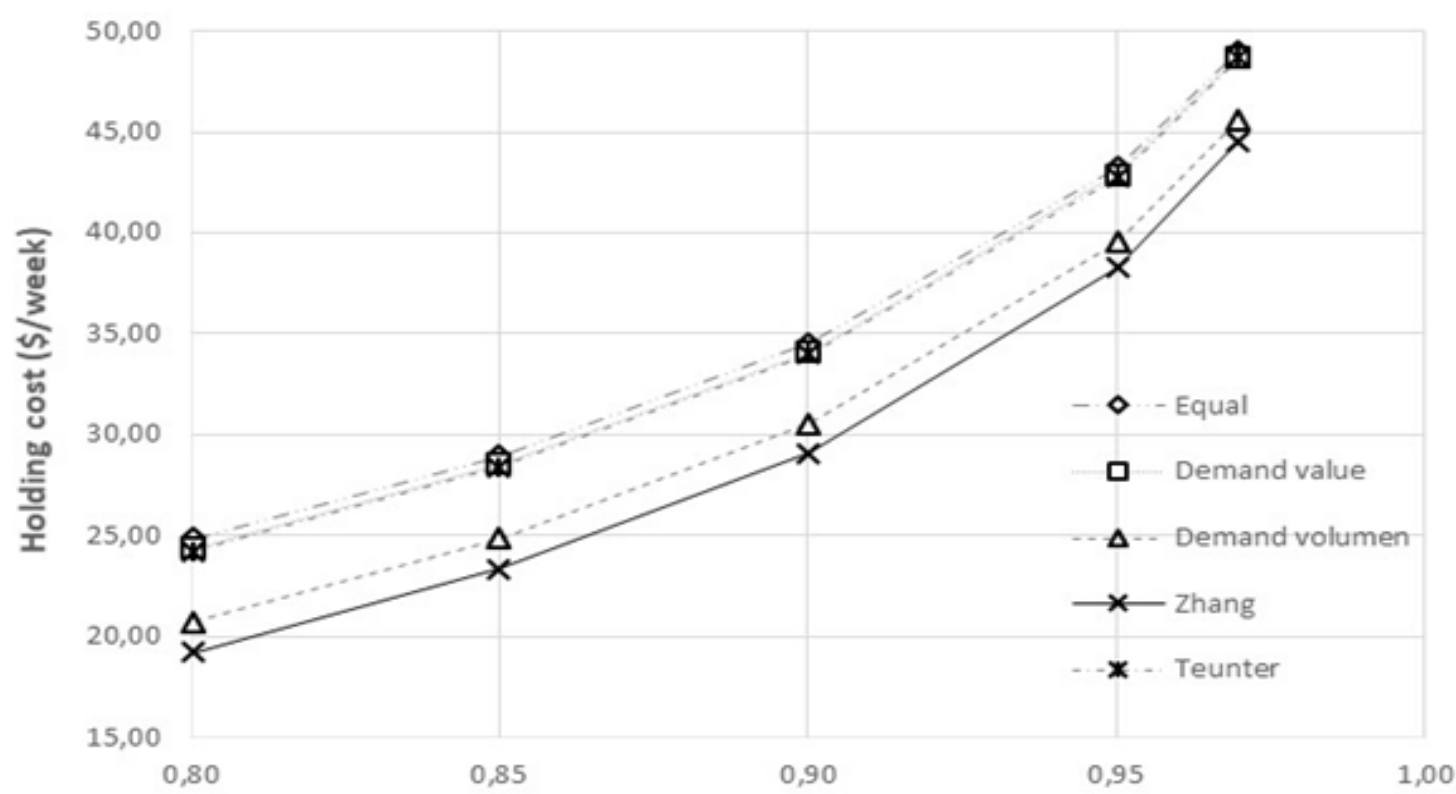

Fill Rate

Figure 2 Cost reduction vs Fill rate

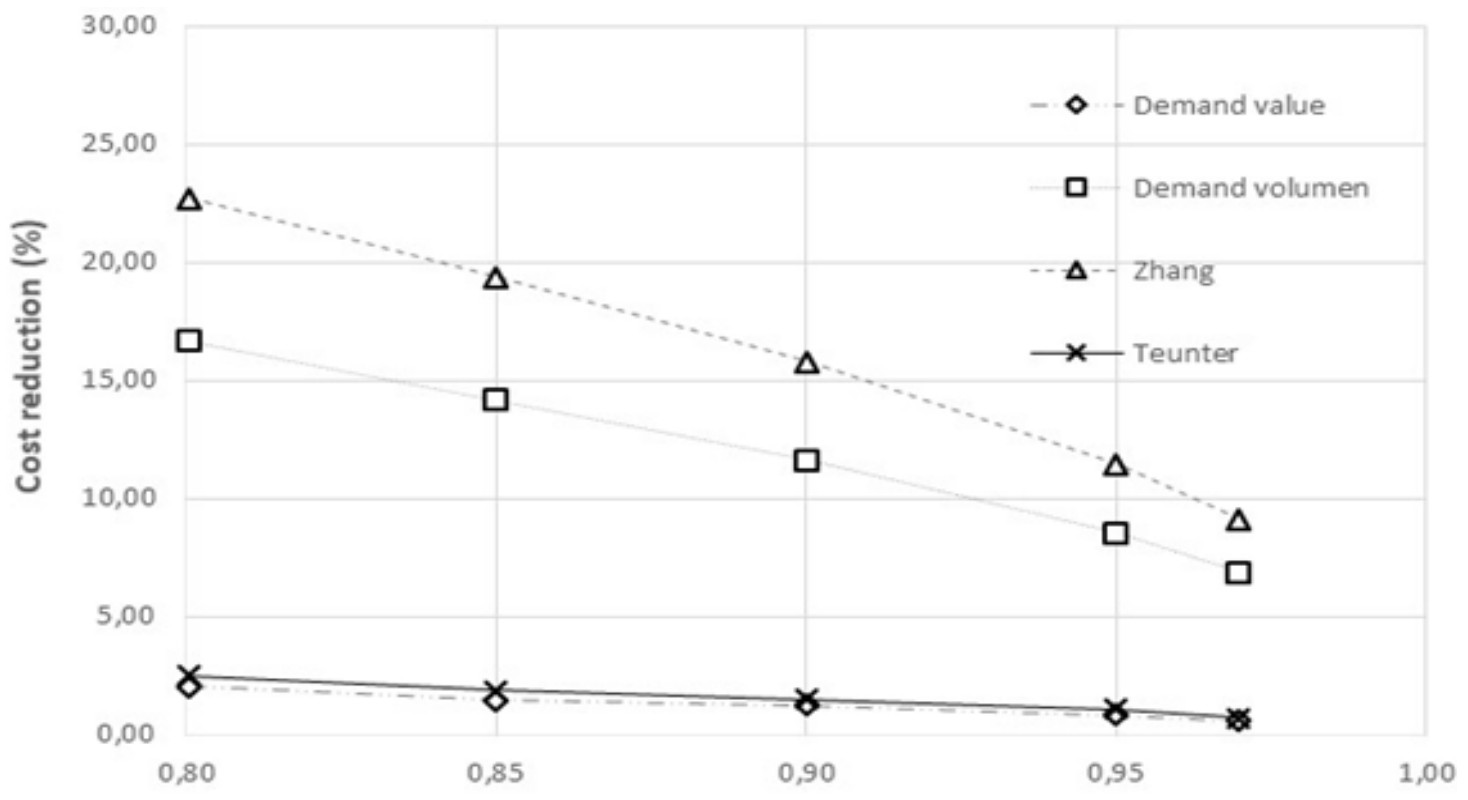

Fill Rate 


\section{A Proposal for Practitioners and Conclusions}

Practitioners need concepts and techniques they can understand and apply without great difficulties, otherwise the technique is usually abandoned. In this respect, the techniques described in section 2.4 are far from being suitable. Another important requirement is the need of a computationally efficient procedure, so the techniques described in section 2.1 approach must also be rejected when thousands of items have to be managed.

Therefore, ABC classification and bidimensional classification are the alternatives left. Based on the literature review and the results reported by authors, following options seem to be the more suitable to be applied by an inventory manager focused on inventory costs and service level. Following options have been ordered by increasing degree of modelling capacity and cost improvement although it also involves more management effort:

1. A simple $\mathrm{ABC}$ analysis based on demand volume ranking because (Teunter, Babai and Syntetos, 2009) showed evidences that this approach provides lower inventory cost than using the economic volume. Our above experiment confirms this statement.

2. An additional cost reduction can be obtained using the Zhang's approach in the ABC classification instead of the unit volume.

3. In case item criticality should be considered, an ABCVED analysis is a good option although considerably more complex. This option can be used with spare parts but also with items for which we want to ensure their availability. However, (Teunter, Babai and Syntetos, 2009) showed that service level decreasing in ABC is more cost effective than the other way round as proposed by (Ramani and Kutty, 1985), so that service levels should decrease in VED and also in ABC.

As a concluding remark, many researches have worked on this problem but more focus is needed on tools and techniques that can be easily applied by inventory managers who pursue minimizing inventory costs while maximizing service levels.

\section{References}

Arcade, S. (1980) 'Characteristics of the Pareto sales curve', Omega, 8(6), pp. 693-694. doi: 10.1016/03050483(80)90011-0.
Arrow, K. J., Harris, T. and Marschak, J. (1951) 'Optimal Inventory Policy’, Econometrica, 19(3), pp. 250-272. Available at: isi:000204900400002.

Babai, M. Z., Ladhari, T. and Lajili, I. (2015) 'On the inventory performance of multi-criteria classification methods: empirical investigation', International Journal of Production Research, 53(1), pp. 279-290. doi: 10.1080/00207543.2014.952791.

Bacchetti, Andrea and Saccani, N. (2012) 'Spare parts classification and demand forecasting for stock control: Investigating the gap between research and practice', Omega, 40(6), pp. 722-737. doi: 10.1016/j. omega.2011.06.008.

Bacchetti, A and Saccani, N. (2012) 'Spare parts classification and demand forecasting for stock control: Investigating the gap between research and practice', Omega-International Journal of Management Science. Univ Brescia, Supply Chain \& Serv Management Res Ctr, Dept Ind \& Mech Engn, I-25123 Brescia, Italy, 40(6), pp. 722-737. Available at: isi:000302990300006.

Bhattacharya, A., Sarkar, B. and Mukherjee, S. K. (2007) 'Distance-based consensus method for ABC analysis', International Journal of Production Research, 45(15), pp. 3405-3420. doi: 10.1080/00207540600847145.

Cardos, M., Guijarro, E. and Babiloni, E. (2015) 'Optimal multi-item fill rate determination for spare parts', in 2015 International Conference on Logistics, Informatics and Service Sciences (LISS). IEEE, pp. 1-5. doi: 10.1109/LISS.2015.7369652.

Cohen, M. A. et al. (1992) 'Multi-Item Service Constrained (s, S) Policies for Spare Parts Logistics Systems', Naval Research Logistics, 39, pp. 561-577.

Cohen, M. A. and Ernst, R. (1988) 'Multi-item classification and generic inventory stock control policies', Production and Inventory Management Journal, 29(3), pp. 6-8.

Ding, B. and Sun, L. (2011) 'An inventory classification model for multiple criteria ABC analysis', 8th International Conference on Service Systems and Service Management - Proceedings of ICSSSM'11. doi: 10.1109/ICSSSM.2011.5959351.

Eilon, S. and Mallya, R. (1985) 'An extension of the classical A B C inventory control system', Omega. Pergamon, 13(5), pp. 429-433. doi: 10.1016/0305-0483(85)900702. 
Flores, B. E., Olson, D. L. and Dorai, V. K. (1992) 'Management of multicriteria inventory classification', Mathematical and Computer Modelling, 16(12), pp. 71-82. doi: 10.1016/0895-7177(92)90021-C.

Flores, B. E. and Whybark, D. C. (1988) 'Implementing multiple criteria ABC analysis', Engineering Costs and Production Economics. Elsevier, 15, pp. 191-195. doi: 10.1016/0167-188X(89)90124-9.

Hadi-Vencheh, A. (2010) 'An improvement to multiple criteria ABC inventory classification', European Journal of Operational Research, 201(3), pp. 962-965. doi: 10.1016/j.ejor.2009.04.013.

Hadi-Vencheh, A. and Mohamadghasemi, A. (2011) 'A fuzzy AHP-DEA approach for multiple criteria ABC inventory classification', Expert Systems with Applications. Elsevier Ltd, 38(4), pp. 3346-3352. doi: 10.1016/j.eswa.2010.08.119.

van Houtum, G. J. and Zijm, W. H. M. (2000) 'On the relation between cost and service models for general inventory systems', Statistica Neerlandica. Eindhoven Univ Technol, Fac Technol Management, NL-5600 MB Eindhoven, Netherlands Univ Twente, Fac Appl Math, NL-7500 AE Enschede, Netherlands, 54(2), pp. 127147. Available at: isi:000090149700003.

van Kampen, T. J., Akkerman, R. and Pieter van Donk, D. (2012) 'SKU classification: a literature review and conceptual framework', International Journal of Operations \& Production Management, 32(7), pp. 850876. doi: 10.1108/01443571211250112.

Kelle, P. (1989) 'Optimal service levels in multi-item inventory systems', Engineering Costs and Production Economics, (15), pp. 375-379.

Lenard, J. D. and Roy, B. (1995) 'Multi-item inventory control: A multicriteria view', European Journal of Operational Research, 87(3), pp. 685-692. doi: 10.1016/0377-2217(95)00239-1.

Mitchell, J. C. (1988) 'Multi-item inventory systems with a service objective', Operations Research, 36(5), pp. $747-755$.

Mohammaditabar, D., Hassan Ghodsypour, S. and Obrien, C. (2012) 'Inventory control system design by integrating inventory classification and policy selection', International Journal of Production Economics. Elsevier, 140(2), pp. 655-659. doi: 10.1016/j.ijpe.2011.03.012.
Molenaers, A. et al. (2012) 'Criticality classification of spare parts: A case study', International Journal of Production Economics. Elsevier, 140(2), pp. 570-578. doi: 10.1016/j.ijpe.2011.08.013.

$\mathrm{Ng}$, W. L. (2007) 'A simple classifier for multiple criteria ABC analysis', European Journal of Operational Research, 177(1), pp. 344-353. doi: 10.1016/j. ejor.2005.11.018

Odanaka, T. and Tanaka, T. (1987) 'On ABC analysis of multiitem inventory problem', Mathematical Modelling. Pergamon, 8, pp. 725-728. doi: 10.1016/02700255(87)90678-6.

Ramanathan, R. (2006) 'ABC inventory classification with multiple-criteria using weighted linear optimization', Computers and Operations Research, 33(3), pp. 695700. doi: 10.1016/j.cor.2004.07.014.

Ramani, S. and Kutty, K. V. K. (1985) 'Management of Multi-Item, Multi-Group Inventories With Multiple Criteria Under Service level constraints', Engineering Costs and Production Economics, 9, pp. 59-64.

Rezaei, J. and Dowlatshahi, S. (2010) 'A rule-based multi-criteria approach to inventory classification', International Journal of Production Research, 48(23), pp. 7107-7126. doi: 10.1080/00207540903348361.

Roda, I. et al. (2012) 'On the classification of spare parts with a multi-criteria perspective', IFAC Proceedings Volumes (IFAC-PapersOnline). IFAC, 45(31), pp. 1924. doi: 10.3182/20121122-2-ES-4026.00020.

Roda, I. et al. (2014) 'A review of multi-criteria classification of spare parts: From literature analysis to industrial evidences', Journal of Manufacturing Technology Management, 25(4), pp. 528-549. doi: $10.1108 / 17410381211267682$.

De Schrijver, S. K., Aghezzaf, E.-H. and Vanmaele, H. (2013) 'Aggregate constrained inventory systems with independent multi-product demand: Control practices and theoretical limitations', International Journal of Production Economics. Elsevier, 143(2), pp. 416-423. doi: 10.1016/j.ijpe.2011.08.019.

Silver, E. A., Pyke, D. F. and Peterson, R. (1998) Inventory Management and Production Planning and Scheduling. John Wiley \& Sons. 
Teunter, R. H., Babai, M. Z. and Syntetos, A. A. (2009) 'ABC Classification: Service Levels and Inventory Costs', Production and Operations Management, 19(3), pp. 343-352. doi: 10.1111/j.1937-5956.2009.01098.x.

Thonemann, U. W., Brown, A. O. and Hausman, W. H. (2002) 'Easy Quantification of Improved Spare Parts Inventory Policies', Management Science. INFORMS, 48(9), pp. 1213-1225. doi: 10.1287/mnsc.48.9.1213.173.

Yu, M. C. (2011) 'Multi-criteria ABC analysis using artificial-intelligence-based classification techniques', Expert Systems with Applications. Elsevier Ltd, 38(4), pp. 3416-3421. doi: 10.1016/j.eswa.2010.08.127.
Zhang, R. Q., Hopp, W. J. and Supatgiat, C. (2001) 'Spreadsheet implementable inventory control for a distribution center', Journal of Heuristics. Cornell Univ, Johnson Grad Sch Management, Ithaca, NY 14853 USA Northwestern Univ, Dept Ind Engn \& Management Sci, Evanston, IL 60208 USA Enron Corp, Res Grp, Houston, TX 77002 USA, 7(2), pp. 185-203. doi: 10.1023/A:1009613921001.

Zheng, S. et al. (2017) 'An improvement to multiple criteria ABC inventory classification using Shannon entropy', Journal of Systems Science and Complexity, 30(4), pp. 857-865. doi: 10.1007/s11424-017-5061-8.

Zhou, P. and Fan, L. (2007) 'A note on multi-criteria $\mathrm{ABC}$ inventory classification using weighted linear optimization', European Journal of Operational Research, 182(3), pp. 1488-1491. doi: 10.1016/j. ejor.2006.08.052. 